Revue

de Sémantique

et Pragmatique

\section{Revue de Sémantique et Pragmatique}

41-42 | 2017

Cadrage sur la variation, le changement lexical et le changement grammatical en français actuel

\title{
Redéfinir l'interjection par la prosodie des actes de langage
}

\section{Chantal Rittaud-Hutinet}

\section{(2) OpenEdition \\ Journals}

Édition électronique

URL : http://journals.openedition.org/rsp/492

DOI : $10.4000 /$ rsp.492

ISSN : 2610-4377

Éditeur

Presses universitaires d'Orléans

Édition imprimée

Date de publication : 1 décembre 2017

Pagination : 225-242

ISSN : $1285-4093$

Référence électronique

Chantal Rittaud-Hutinet, «Redéfinir l'interjection par la prosodie des actes de langage ", Revue de Sémantique et Pragmatique [En ligne], 41-42 | 2017, mis en ligne le 01 décembre 2018, consulté le 04 mai 2019. URL : http://journals.openedition.org/rsp/492 ; DOl : 10.4000/rsp.492 
erroger

ici avec vous : l'interjection. Par l'analyse de quelques-unes de ses dimensions orales, mon objectif est de montrer et de démontrer l'impact de cette troisième articulation du langage qu'est la prosodie signifiante sur ce qui est compris par 
ernet. Puis je procéderai au décryptage des signifiés de quelques 
Redéfinir l'interjection par la prosodie des actes de langage

avent amplifier ou contredire, détourner, rendre ridicule, manifes

à le mettre en difficulté, à lui prendre la parole, à l'empêcher de contester

$\operatorname{sim}$

niste en cas

ssé quelques études 
ppelons, enfin, que chaque signifiant est associé à un signifié et à un seul, ce qui signifie que le complexe d'indices acoustiques 
e, les interjections - comme les onomatopées - posent de vrais problèmes. On lit par exemple chez Josette Rey-Debove qu'il «règne une grande confusion dans les dictionnaires à ce sujet /et que/ ce sont des mots entièrement sacrifiés aussi bien dans la macrostructure que dans la microstructure»

e «souvent on confond l'onomatopée avec les mimologismes, les huchements et les interjections. /.../L' analyse est souvent insuffisante. On peine déjà à leur trouver un nom /... et/ les indications de catégories grammaticales éclatent dans de multiples directions quand il ne s'agit pas de bruits»

e traitant pas les interjections à partir de leurs nombreuses réalités orales. Ce que je m'efforcerai de faire.

\subsection{QUELQUES DÉFINITIONS ET «VALEURS»}

Mais jetons d'abord un coup d'œil aux usages annoncés (repris dans le

isé sur la fonction ;

us

ion grammaticale.» L'affirmation semble contredite plus loin par : «On classe aussi parmi les interjections des mots-phrases». Que doit-on com

ttp://la-conjugaison.nouvelobs.com 
* «La classe des interjections comprend des mots servant à marquer une réaction, un sentiment, une sensation ou une émotion», un peu plus loin : «ils servent à donner des ordres» et, encore plus loin : «Les jurons et les injures en font également partie». Enfin, dans la remarque finale : «Exemple :

gacement, etc.». Point positif, la liste des emplois est plus étof

orale, l'émotion étant souvent exprimée par l'intonation», attaque enfin le véritable sujet, même si on y parle de «nuances» et non de signifiés différents d'un cas à l'autre, et même si le phénomène est annon

$\mathrm{n}$, dont vous pouvez lire les extraits dans le tableau

re un bruit, un cri». Cette définition initiale

'exprimer que fortement... On

e un accent d'intensité dans le

discours oral»; et à la fin de la remarque 4 (la dernière) : «La valeur

tiels pour notre

propos, car : le terme «intonation» recouvre sans doute l'ensemble de

e»

de la définition initiale. Mais il ne s'agit que d'une seule des possibilités sonores exploitées par les interjections.

* Troisièmement, on se rend compte que la plus grande partie des «autres remarques»

ement comme des mots «invariables, 
cris ou des onomatopées ; b) des substantifs accom

ines interjections peuvent avoir des compléments.

2. Si l'interjection est généralement invariable, on rencontre quelques

s l'écriture».

et donc de la classi

hrase /.../ Mais l'inter

re l'interjection et l'onomatopée

'interjection en 3

ignation, commentaire, aparté, aveu, valeur appréciative, désengagement, contestation, rectification, implica

tion». Cet ensemble déjà conséquent est encore suivi d'une remarque, qui ressemble à un résumé, je cite : «/oh/ marque une

». Enfin, cette remarque est elle-même suivie

oh», la définition du groupe 1 - sur 3 groupes fait état de la prosodie : «dans la langue parlée et dans le discours écrit qui la reflète, /ah/ sert à marquer, avec l'aide d'intonations appropriées (que l'écriture 
; et enfin

«la surprise, l'admiration, l'enthousiasme». Dans les deux autres groupes sont également signalées des modalisations : «/ah/ ne sert souvent qu'à donner un tour plus énergique à la phrase ou à introduire une idée nouvelle»; «redoublée, exprime la surprise, l'ironie, la satisfaction».

\begin{tabular}{|l|l|l|l|l|}
\hline & & & & \\
\hline & & & & \\
\hline \\
\hline
\end{tabular}

t porter, j'ai choisi 3 types d'exemples :

- Le premier est tiré d'un film de 1937 vu à la télévision,

bert Seller, jouant le 
Redéfinir l'interjection par la prosodie des actes de langage

téléphone chez sa fille pour prendre de ses nouvelles, car cette dernière et

rancis est seul à l'écran/ Aaah ! C'est Madame ! Madame est de retour ! Ah j(e) suis content d'entendre Madame ! J'ai bien

pondant/ ((silence)) Oh ! ((silence)) Oh !!

sans autre précision, de «jouer» à sa guise les

rire ce que signifiaient pour eux les items. Ce test interprétatif m'a permis de vérifier la concordance des explications entre les informateurs, bien qu'avec des formulations diverses. Ces informations confirment en outre la va

ha ha !» puis : «o aha !»

$\mathrm{rd}:$ « ha 
n», comme l'écrivait C. Blanche-Ben

onctuation on observe quelques figures, à savoir le point d'interrogation (?) celui d'admiration ou d'exclamation (!) \& les points de réticence (...) qui marquent dans ces occasions la difference

t parlant - sinon impossible - de trouver les mots correspondant à son objectif, avec la complexité souhaitée

j'ai utilisé soit le discours direct, en quelque sorte la «réplique verbale» de ce que tente de transmettre l'infor

s peur !»

drée»

is : la capacité de ce coffre est ef

le, quelle découverte extraordi

ent ?» 
Redéfinir l'interjection par la prosodie des actes de langage

fant : «C'est très mal, ce que tu as fait !! Je suis très fâché !»

voir/de constater ça !»

i j'avais su...»

xtension formelle peut aller d'une

s sur la dernière syllabe 
u'à un seul signifié ;

n ou de diminution progressive ; 4 enfin appa

stiques et de leurs combinaisons spécifiques 
Redéfinir l'interjection par la prosodie des actes de langage 
s'agit d'apprendre les règles par cœur - c'était encore le cas quand mes fils allaient à l'école

ibués par les écrits à notre

ser les interjections à bon es

ui régissent les emplois, mais bien par la pratique orale en situation; ensuite, parce que nous employons des interjections

$r$ changer la donne et arriver à décrire efficace 
Redéfinir l'interjection par la prosodie des actes de langage

pkossan, Johanne \& Delumeau, Fabrice (2012), « Comment la prosodie donne du sens aux interjections?», 


\section{jamins}

Blanche-Benveniste, Claire (1991), «Les études sur l'oral et le travail d'écriture de certains poètes contemporains »,

ique (2003) : « La focalisation en français : morphologie de la prosodie », in Aubergé V., Lacheret-Dujour A. \& Lœvenbruck H.

REST Expressive Speech Project, 67-74

Carston Robyn (2009), « The explicit/implicit distinction in pragmatics and the limits of explicit communication »,

spers, Johanneke, Chen, Yiya, Heeren, Willemijn \& Pacilly, Jos (

approche de la «distribution » et des fonctions en discours»,

de consensua

rent (2002), « Transcrire les données vocales : en quoi les corrélats proso 
de chat », communication au colloque international du CeReS : « Autour des formes implicites », Limoges 13-14 novembre (

s marqueurs discursifs et intersubjectivité : autour de

e Gac, David \& Yoo, Hiyon (2009), «Les primitives intonatives du français : une proposition», in

y-Annick (1995), «Valeur énonciative des variations de hauteur mélodique en français»,

ntention»,

ud-Hutinet, Chantal (2010), «Quand les écrivains parlent linguistique», in Ablali D. \& Kastberg Sjöblom M.

t variation du sens», in Bertrand O. \& 
au colloque inter

vocaux ?», à paraître (2016) in

vent les signes

1 (2016b), «Comment comprendre les non-dits ?», in Castagne 